\title{
Vinpocetine Inhibits Oligodendroglial Precursor Cell Differentiation
}

\author{
Klintsy Julieta Torres ${ }^{1}$ Peter Göttle ${ }^{1} \quad$ David Kremer ${ }^{1} \quad$ Jose Flores Rivera ${ }^{2}$ Lucinda \\ Aguirre-Cruz $^{2}$ Teresa Corona ${ }^{2}$ Hans-Peter Hartung ${ }^{1}$ Patrick Küry ${ }^{1}$
}

${ }^{1}$ Heinrich-Heine-University, Medical Faculty, Department of Neurology, Düsseldorf, Germany; ${ }^{2}$ National Institute of Neurology and Neurosurgery, La Fama-Tlalpan, Mexico D. F, Mexico

\section{Key Words}

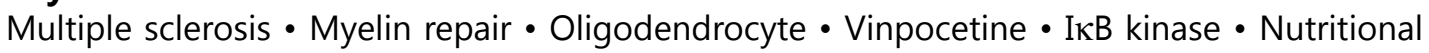
supplement

\begin{abstract}
Background: In multiple sclerosis during periods of remission a limited degree of myelin repair can be observed mediated by oligodendroglial precursor cells. Phosphodiesterase inhibitors act as anti-inflammatory agents and might hold promise for future multiple sclerosis treatment. Aims: To investigate whether phosphodiesterase inhibitors could also influence myelin repair. Methods: We stimulated primary oligodendroglial precursor cells with cilostazol, rolipram and vinpocetine and assessed their effects on repair related cellular processes. Results: We found that vinpocetine exerted a strong negative effect on myelin expression while cilostazol and rolipram did not show such effects. In addition, vinpocetine decreased morphological complexities suggesting an overall negative impact on oligodendroglial cell maturation. We provide evidence that this is not mediated via a blockade of phosphodiesterase- 1 but rather by inhibition of IкB kinase. Conclusion: These findings suggest that vinpocetine via IKB inhibition exerts a strong negative impact on oligodendroglial cell maturation and may therefore provide the rationale to restrict its application during periods of remission in multiple sclerosis patients. This is of particular interest since vinpocetine is widely used as a health supplement thought to act as a cognitive and memory enhancer for healthy people and patients with neurological or muscle diseases.
\end{abstract}




\section{Introduction}

Multiple sclerosis (MS) is the most common demyelinating disease of the western hemisphere featuring loss of oligodendrocytes and myelin sheaths as a consequence of autoimmune attacks on the central nervous system (CNS). Current treatments focus on the prevention of immune cell infiltration and a number of novel immunomodulatory agents have recently been approved or are in late-stage development for relapsing/remitting MS [1]. Despite the generally low regenerative capacity of the adult CNS, a certain degree of myelin repair can be observed based on activation, recruitment and differentiation of either resident oligodendroglial precursor- (OPCs; $[2,3]$ ) or neural stem cell populations (NSCs; $[4,5])$. Remyelination, however, remains inefficient due to differentiation blockade [6]. In this regard a number of negative regulators have recently been described which influence these cells' capacity to differentiate and mature (reviewed in [7]). Since immunomodulatory treatments may provide adequate salvage from novel attacks future therapeutic approaches must be designed in order to improve neuroprotection and tissue repair, including restoration of myelin sheaths.

Cyclic nucleotides such cGMP and cAMP act as second messengers and have been implicated in a variety of physiological functions including gene regulation, cellular differentiation and immunomodulation [8]. Eleven different families of phosphodiesterases (PDEs) have been described, enzymes that regulate cytoplasmic cAMP and cGMP levels by hydrolyzing them into 5 'monophosphates, therefore turning off signaling cascades. PDE inhibitors have been used as anti-inflammatory agents for the treatment of different brain diseases [9], while cilostazol (CTZ), a PDE3 inhibitor [10], and rolipram (RLP), a PDE4 inhibitor [11], have been used for experimental autoimmune encephalomyelitis (EAE) treatment. Vinpocetine (VPN), a PDE1 inhibitor and derivative from vincamine was shown to exert a strong anti-inflammatory effect on HUVEC cells $[12,13]$ and is used as treatment for cerebrovascular diseases [14]. In addition, it is widely used as "memory and cognitive enhancer", and can be purchased without medical prescription as alimentary supplement in many countries [15]. Vinpocetine is also commercially promoted for many brain diseases (including MS) supposed to significantly increase cerebral blood flow, probably through vasodilation. Of note, for several PDE inhibitors used in rodent EAE models, an amelioration of clinical symptoms could be observed [16-18]. Likewise, administration of rolipram prevented the demyelination process in non-human primates [19]. However, it is currently unclear whether apart from effects on cytokine production and T cell response [20, 21], PDE inhibitors are also able to affect regenerative processes such as myelin repair.

This study aimed at elucidating a potential role of PDE inhibitors in modulating myelin repair by influencing differentiation processes of oligodendroglial precursor cells. Our results provide evidence that vinpocetine has the ability to block spontaneous as well as chemokinepromoted OPC maturation, a function which was found to be related to the inhibition of IKK (inhibitor of nuclear factor kappa-B kinase), rather than to modulation of cyclic nucleotide concentrations.

\section{Materials and Methods}

\section{Oligodendroglial precursor cell culture}

Primary OPCs were obtained from postnatal day zero (P0) cerebral rat cortices as previously described [22, 23]. Anti-A2B5 staining (data not shown) revealed that the cultures consisted of 98\% oligodendroglial cells. OPCs were either kept in proliferation medium [high glucose DMEM-based Sato medium (Life Technologies, Darmstadt, Germany) supplemented with $10 \mathrm{ng} / \mathrm{ml}$ recombinant human basic fibroblast growth factor (bFGF; PeproTech, Hamburg, Germany) and 10ng/ml recombinant human PDGF-AA; R\&D Systems, Wiesbaden-Nordenstadt, Germany)] whereas differentiation was initiated by Sato medium supplemented with $0.5 \%$ fetal calf serum (FCS; PAA, Linz, Austria). PDE and IKK inhibitors cilostazol (CTZ; 6-[4-(1-cyclohexyl-1H-tetrazol-5-yl)-butoxy]-3,4-dihydro-2(1H)-quinolinone; Sigma- 
Torres/Göttle/Kremer et al.: IKB Kinase and Oligodendrocyte Maturation

Aldrich, St. Louis, MO), rolipram (RLP; 4-[3-(cyclopentyloxy)-4-methoxyphenyl]-2-pyrrolidinone; SigmaAldrich), vinpocetine (VPN; $(3 \alpha, 16 \alpha)$-eburnamenine-14-carboxylic acid ethyl ester; Sigma-Aldrich), MIMX (8-methoxymethyl-3-isobutyl-1-methylxanthine; Sigma-Aldrich), IKK inhibitor III (4-(2'-aminoethyl)amino1,8-dimethylimidazo[1,2-a]quinoxaline; Merck, Darmstadt, Germany) and IKK inhibitor VII (benzamidopyrimidine; Merck) were all resuspended in ethanol. Optimal concentrations of PDE and IKK inhibitors were obtained according to literature and using dose response curves (data not shown) and finally applied as $10 \mu \mathrm{M}$ VPN, $5 \mu \mathrm{M}$ CTZ, $10 \mu \mathrm{M}$ RLP, $0.5 \mu \mathrm{M}$ IKK inhibitor III, $0.25 \mu \mathrm{M}$ IKK inhibitor VII, and up to $250 \mu \mathrm{M}$ MIMX. Control cells were treated with identical ethanol dilutions, medium was changed every three days. Recombinant human CXCL12 (R\&D Systems) stimulation was carried out at a concentration of $100 \mathrm{ng} / \mathrm{ml}$.

\section{Immunofluorescence stainings and morphological assessment}

Immunofluorescence stainings on $4 \%$ paraformaldehyde-fixed cultured cells were performed as described previously $[22,23]$. Primary antibodies were diluted as follows: mouse anti-04- and mouse antiA2B5 antibodies (1/100,1/200, respectively; both Millipore, Schwalbach, Germany), anti-MOG antibody (1/1000; B. Hemmer), anti-MBP- and anti-CNPase antibodies (1/1000 and 1/500, respectively; Covance, Princeton, USA) and rabbit anti-caspase3 (1:500, Cell Signaling Technology, Denver, USA). Alexa Fluor 488and Alexa Fluor 594-conjugated antibodies (1/500; Molecular Probes, Leiden, The Netherlands) were used for signal visualization. Nuclei were stained with 4',6-diamidino-2-phenylindol (DAPI; Roche, Basel, Switzerland). 04 staining was used to evaluate morphological cell parameters (processes, branches) and we distinguished three different morphologies from a very low number of processes in progenitor cells ("early") to multiple process-bearing ("intermediate") cells and "late" stages with flattened appearance. Marker expression was analyzed by fluorescence microscopy (Zeiss Axioplan, Jena, Germany), and analysis was done using ImageJ software (NIH), counting nine fields per coverslip and in duplicates. Each experiment was performed at least three times and data shown in Figs. 2, 3, 4, 6 derived from one representative experiment each [mean values $+/$ - standard error of mean (SEM)] and statistical significance was calculated by Student's t-test.

RNA preparation, cDNA synthesis, and real-time quantitative RT-PCR

Total RNA purification was done using the RNeasy procedure (Qiagen, Hilden, Germany). RNA was reverse transcribed using the high-capacity cDNA Reverse Transcription Kit (Applied Biosystems, Darmstadt, Germany). Quantitative determination of gene expression levels was performed on a 7900HT sequence detection system (Applied Biosystems) using Power SybrGreen Universal Master Mix (Applied Biosystems). Primer sequences were determined using PrimerExpress 2.0 software (Applied Biosystems) and tested for the generation of specific amplicons:

CNPase_fwd: ATGCTGAGCTTGGCGAAGAA,

CNPase_rev: GTACCCCGTGAAGATGGCC, MBP_fwd: CAATGGACCCGACAGGAAAC, MBP_rev: TGGCATCTCCAGCGTGTTC, MOG_fwd: CAGTTGTCACGCAGCTACGC, MOG_rev: ATGCCCTGGCCCTATCACTC, PLP_fwd: CTTTGGAGCGGGTGTGTCAT, PLP_rev: TGTCGGGATGTCCTAGCCAT, Hes1_fwd: GGAGAGGCTGCCAAGGTTTT, Hes1_rev: AAGCAAATTGGCCGTCAGG, Id2_fwd: AGAACCAAACGTCCAGGACG, Id2_rev: TGCTGATGTCCGTGTTCAGG, Id4_fwd: CAGCTGCAGGTCCAGGATGT, Id4_rev: AAAGTGGAGATCCTGCAGCAC, p57kip2.1_fwd: CAGGACGAGAATCAGGAGCTGA, p57kip2.1_rev: TTGGCGAAGAAGTCGTTCG, Rock2_fwd: GATGGTTACTATGGACGAGAATGTGA, Rock2_rev: AGTGTCCCCTACTAACATCTCAAAAAG, GAPDH_fwd: GAACGGGAAGCTCACTGGC, GAPDH_rev: GCATGTCAGATCCACAACGG 


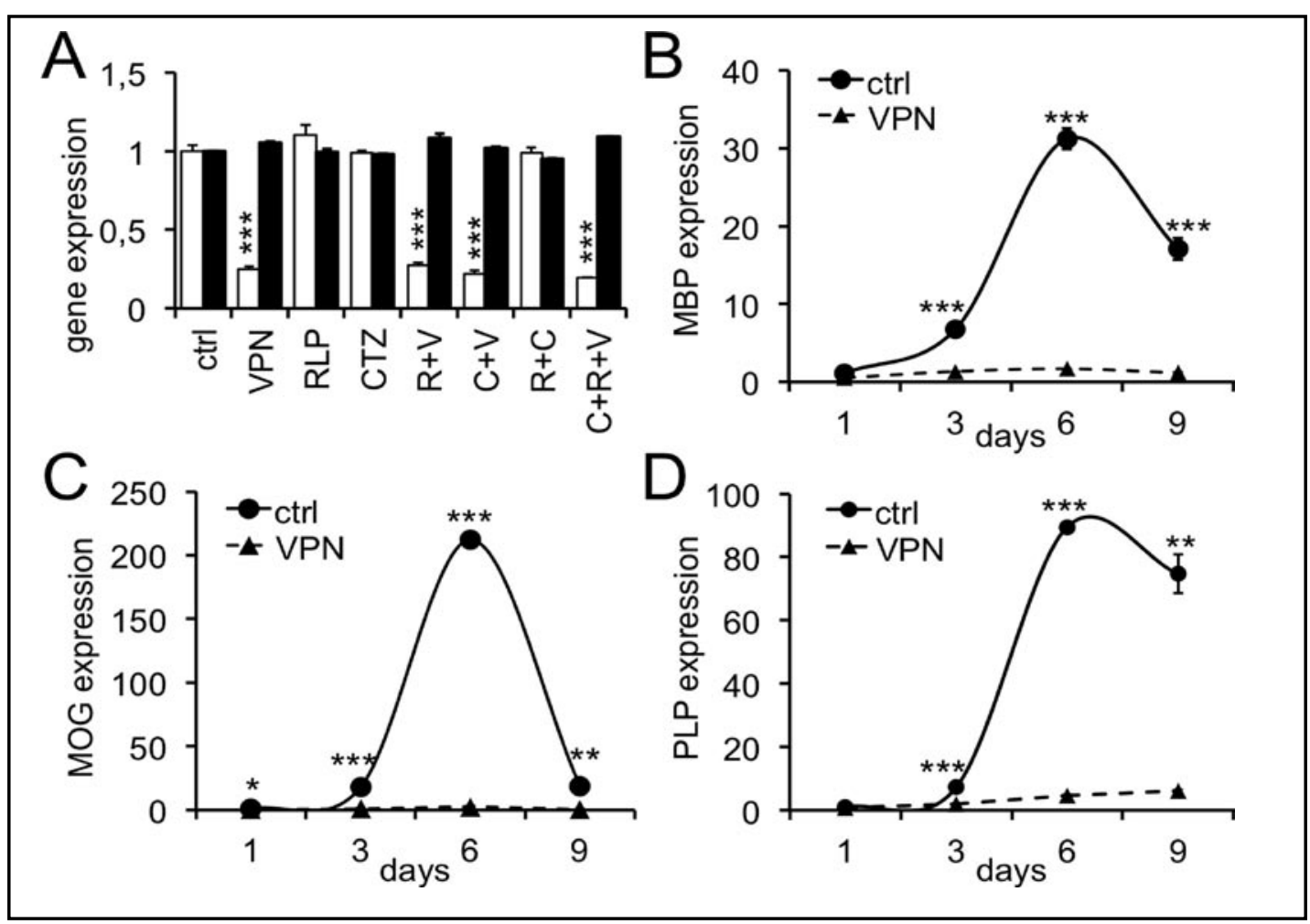

Fig. 1. Regulation of myelin gene expression in oligodendroglial precursor cells. (A) OPCs were treated with vinpocetine (VPN or V), cilostazol (CTZ or $\mathrm{C}$ ), rolipram (RLP or $\mathrm{R}$ ) and combinations $(\mathrm{R}+\mathrm{V}$; $\mathrm{C}+\mathrm{V}$; $\mathrm{R}+\mathrm{C} ; \mathrm{C}+\mathrm{R}+\mathrm{V}$ ) revealing that only vinpocetine suppressed MBP gene expression (white bars) after $48 \mathrm{hrs}$ of treatment. Note that these substances did not affect CNPase (black bars) expression levels. (B-D) Time course stimulation experiments demonstrating that VPN suppressed MBP, MOG and PLP expression up to nine days of treatment. Transcript levels were determined by real-time polymerase qRT-PCR; GAPDH expression was used as reference. One representative out of two independent measurements is shown for each determination. Data are mean values +/- SEM; Student's t-test of treatment vs. control at any time point: ${ }^{* *} \mathrm{p}<0.01,{ }^{* * *} \mathrm{p}<0.001$.

Glyceraldehyde-3-phosphate dehydrogenase (GAPDH) was used as reference gene and relative gene expression levels were determined according to the $\Delta \Delta \mathrm{Ct}$ method (Applied Biosystems). Each sample was measured in quadruplicate, data are shown as mean values +/- SEM and Student's t-test was applied to determine statistical significance.

\section{Results}

\section{Vinpocetine blocks myelin expression}

Since PDE inhibitors have been shown to either ameliorate EAE symptoms [10,11], or are evaluated for future MS treatments $[9,24]$ we wondered whether these substances could also modulate myelin repair. We therefore investigated their effects on cultured primary OPC differentiation and treated these cells during 48 hours with $10 \mu \mathrm{M}$ VPN, $5 \mu \mathrm{M}$ CTZ and 10 $\mu \mathrm{M}$ RLP as well as with combinations of two or three PDE inhibitors. Transcript levels of the early myelin gene 2',3'-cyclic nucleotide 3'-phosphodiesterase (CNPase) and the myelin basic protein (MBP) were determined using real-time quantitative reverse transcription polymerase chain reaction (qRT-PCR) which revealed a strong downregulation of MBP expression on VPN treated cells but not upon stimulation with CTZ or RLP (Fig. 1A). CNPase 


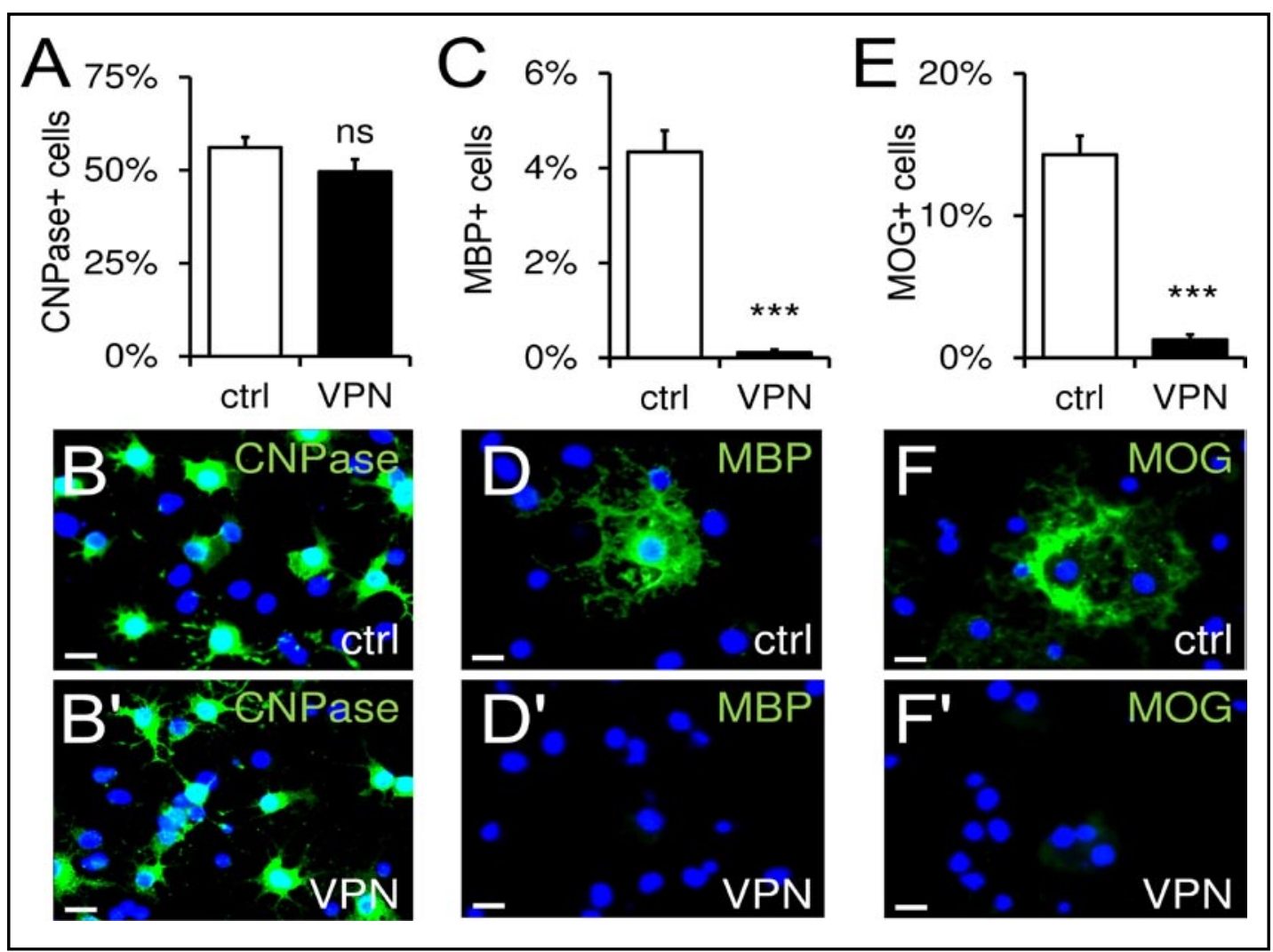

Fig. 2. Expression of myelin proteins CNPase, MBP and MOG in oligodendroglial precursor cells. Quantitative determination of the degree of (A) CNPase-positive cells after one day of VPN treatment, (C) MBP-positive cells after three days of VPN treatment and (E) of MOG-positive cells after six days of VPN stimulation. Data are mean values +/- SEM and derive from a single representative experiment out of three experiments (Student's t-test: ns: not significant, $\left.{ }^{* * *} \mathrm{p}<0.001\right)$. (B, B', D, D', F, F') Representative images of immunostained control and VPN stimulated OPCs (blue color: DAPI staining, scale bars $10 \mu \mathrm{m}$ ).

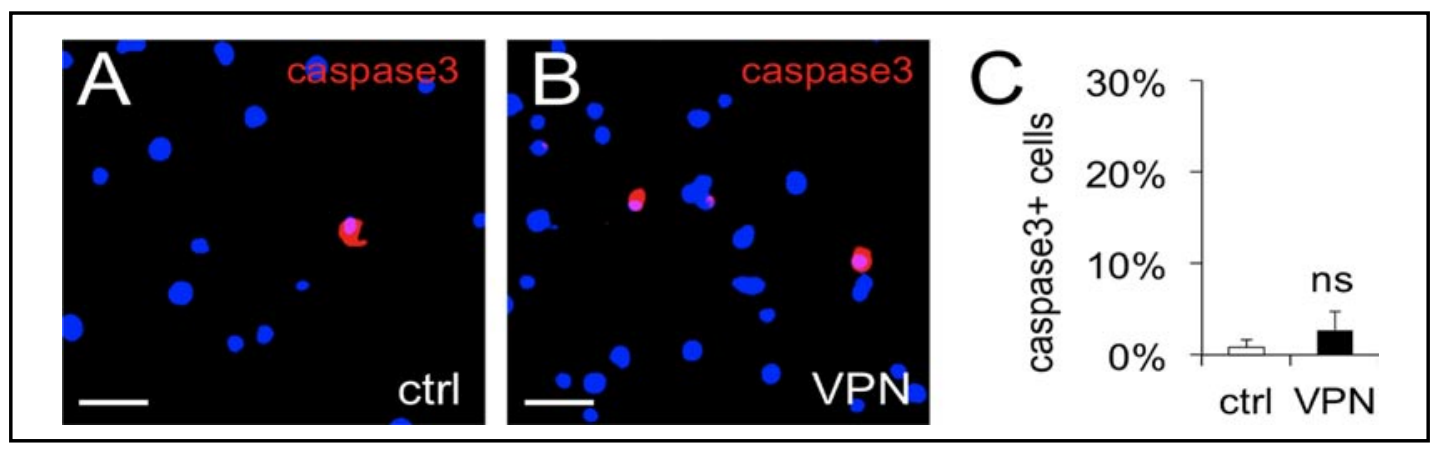

Fig. 3. Oligodendroglial programmed cell death. In order to rule out a VPN dependent effect on cell survival we performed stainings using anti-caspase 3 antibodies (A, B). No significant difference in the frequency of apoptotic cells among control and VPN treated cells was detected (C). (Student's t-test of treated vs. control OPCs; ns: not significant). Scale bars $20 \mu \mathrm{m}$.

expression was not affected by these substances (Fig. 1A). Time course experiments on OPCs treated for one, three, six and nine days with VPN demonstrated a long lasting and strong downregulation of MBP (Fig. 1B) but also of myelin oligodendrocyte glycoprotein (MOG; Fig. 1C) and proteolipid protein (PLP; Fig. 1D) gene expression. The expression of the early myelin gene 2',3'-cyclic nucleotide 3'-phosphodiesterase (CNPase) was not affected 


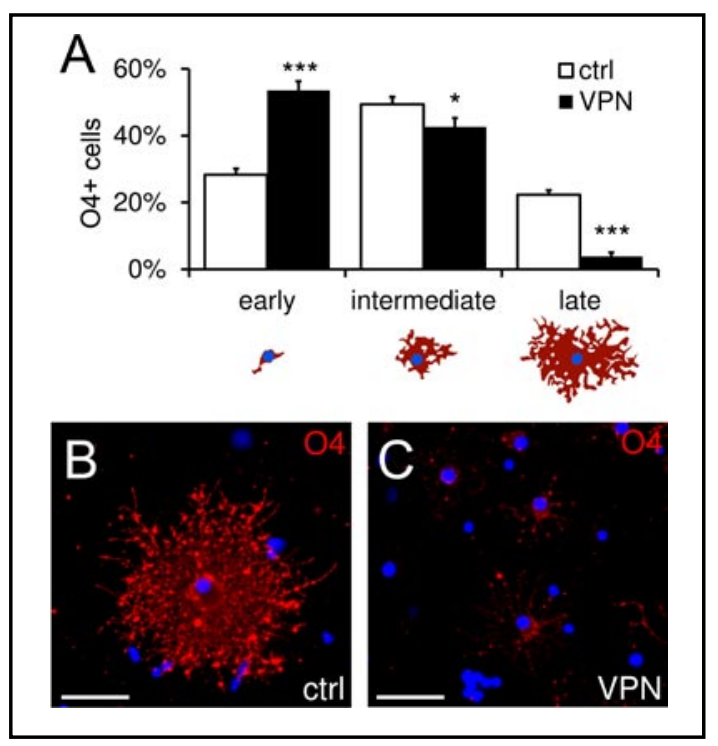

Fig. 4. Morphology of oligodendroglial precursor cells. 04 immunostaining was performed in order to visualize cells and cellular processes. (A) Quantification of OPCs with early, intermediate or late cellular morphologies revealed that upon treatment with $10 \mu \mathrm{M}$ VPN for three days the number of early stage OPCs was significantly increased, whereas intermediate and late stages were significantly reduced. Data are mean values +/- SEM (Student's t-test: $\left.{ }^{*} \mathrm{p}<0.05,{ }^{* * *} \mathrm{p}<0.001\right)$ and derive from a single representative experiment out of three experiments. (B, C) Representative 04 stained cells under control conditions and upon VPN stimulation (scale bars $50 \mu \mathrm{m}$ ).

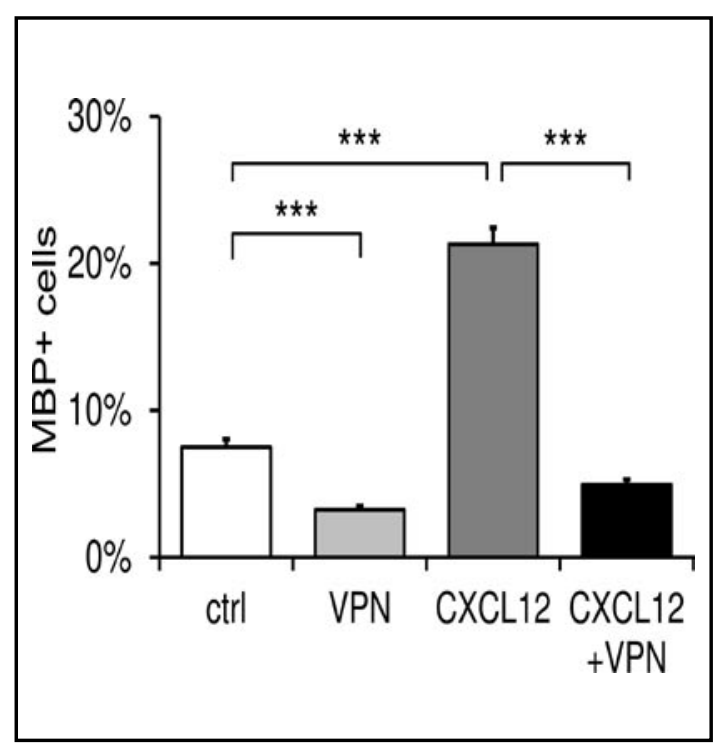

Fig. 5. Chemokine dependent promotion of oligodendroglial differentiation. OPCs were treated with $10 \mu \mathrm{M}$ VPN and/or $100 \mathrm{ng} / \mathrm{ml}$ CXCL12 for three days and the degree of MBP-positive cells was determined quantitatively. VPN reduced the number of MBP expressing cells under control conditions and could efficiently counteract the CXCL12 dependent induction of MBP positivity. Data are mean values $+/$ - SEM (Student's t-test: ${ }^{* * *} \mathrm{p}<0.001$ ) and derive from a single representative experiment out of three experiments.

(data not shown). We next determined whether VPN also downregulated myelin protein expression. Control and VPN treated OPCs were fixed and processed for immunofluorescence stainings. The number of myelin positive cells was determined and this demonstrated that while CNPase positivity ( 24 hours; Fig. 2A, B) was not affected by VPN, degrees of MBP(three days of treatment; Fig. 2C, D) and MOG-expressing cells (six days of treatment; Fig. 2E, F) were strongly decreased by VPN as compared to controls. In order to rule out a VPN dependent effect on cell survival we performed stainings using anti-caspase 3 antibodies. No significant difference in the frequency of apoptotic cells among control and VPN treated cells was noted (Fig. 3) indicating that the observed differences in gene and protein expression were not based on alterations in cell survival. Since in our culture system spontaneous OPC differentiation is initiated following mitogen withdrawal [22], VPN dependent effects on cell proliferation could be excluded.

\section{Vinpocetine disturbs morphological maturation of OPCs}

Oligodendroglial differentiation is a combination of gene/protein regulatory events and alterations in cell size and shape. However, morphological maturation of cultured OPCs is not synchronized and is seen as a heterogeneous cell population with various degrees of maturation, featuring increasing numbers of processes and branches. We therefore determined quantitatively whether OPC maturation is influenced by VPN among 04 


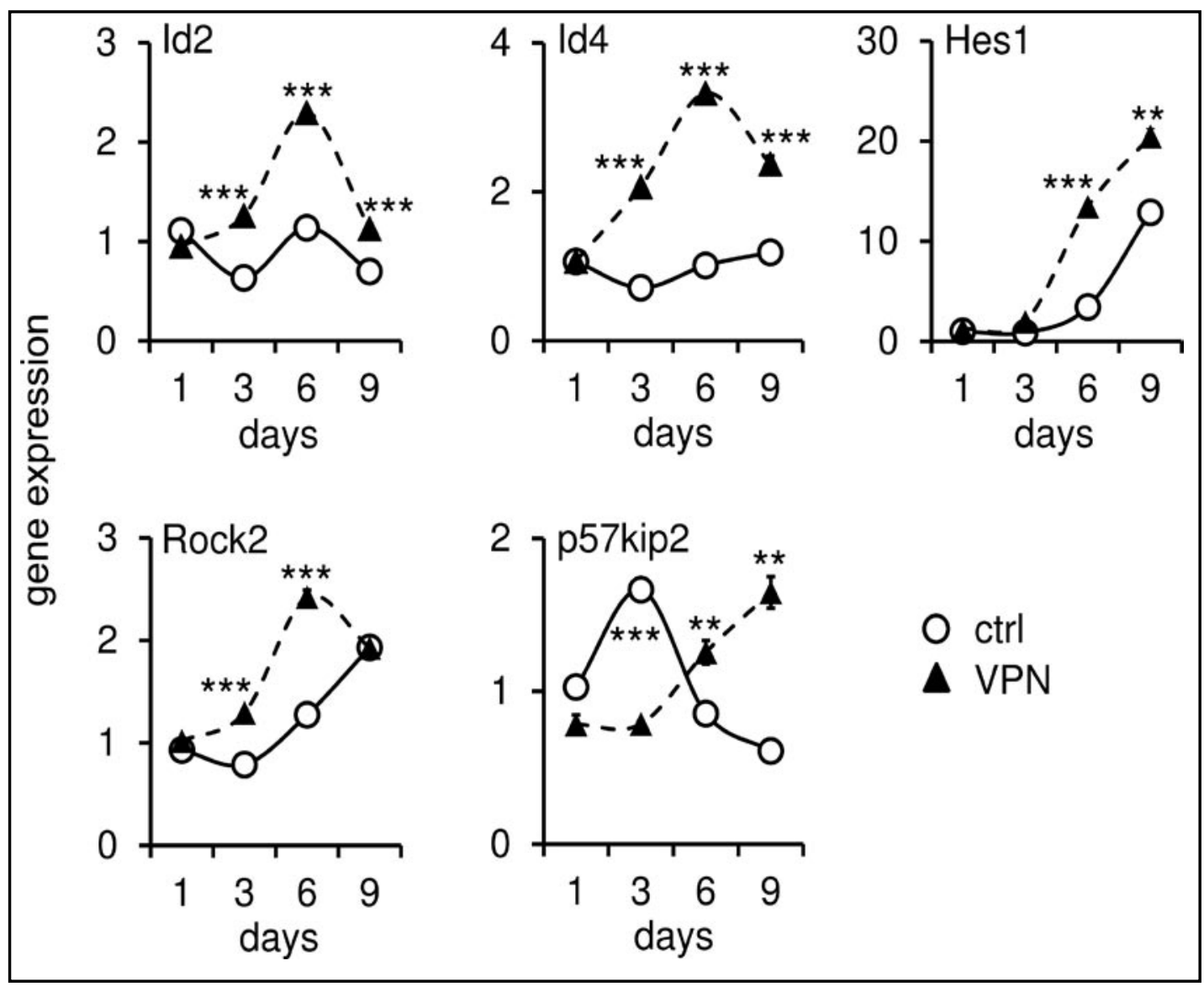

Fig. 6. Temporal expression analysis of genes encoding oligodendroglial differentiation inhibitors. Determination of transcript levels by real-time quantitative RT-PCR demonstrated that expression of Id2, Id4, Rock2 and Hes1 were significantly elevated throughout the whole stimulation procedure $(1,3,6$ and 9 days, respectively) and that the biphasic expression pattern of p57kip2 was altered leading to increased expression levels at late time points. GAPDH expression was used as reference. One representative out of three independent measurements is shown; data are mean values +/- SEM (Student's t-test of treatment vs. control at any time point: ${ }^{* *} \mathrm{p}<0.01 ;{ }^{* * *} \mathrm{p}<0.001$ ).

expressing cells (Fig. 4) given that the 04 sulfatide is expressed during almost the entire OPC differentiation process [25]. OPCs were treated for three days with VPN or control buffer, fixed, stained using an anti-04 antibody and classified in three maturation stages (early, intermediate and late). Quantitative analysis of the distribution of these three stages among 04-positive cells revealed a significant delay in the maturation pattern upon stimulation with VPN featuring an increase in the number of early stage- and decreased degrees of intermediate and late stage morphologies.

\section{Vinpocetine counteracts CXCL12 mediated promotion of OPC differentiation}

We could recently demonstrate that the chemokine CXCL12 activates the CXCR7 receptor on OPCs and that this interaction strongly promotes oligodendroglial differentiation [23]. In order to determine if VPN could counteract this strong positive regulator, co-stimulation experiments were conducted and the degree of MBP-positive cells was determined (Fig. 5). OPCs were treated during three days with $100 \mathrm{ng} / \mathrm{ml} \mathrm{CXCL12} \mathrm{and/or} 10 \mu \mathrm{M}$ vinpocetine (VPN) or control buffer. This clearly demonstrated that a single treatment with VPN significantly decreased the number of MBP-positive cells in both, absence as well as presence 


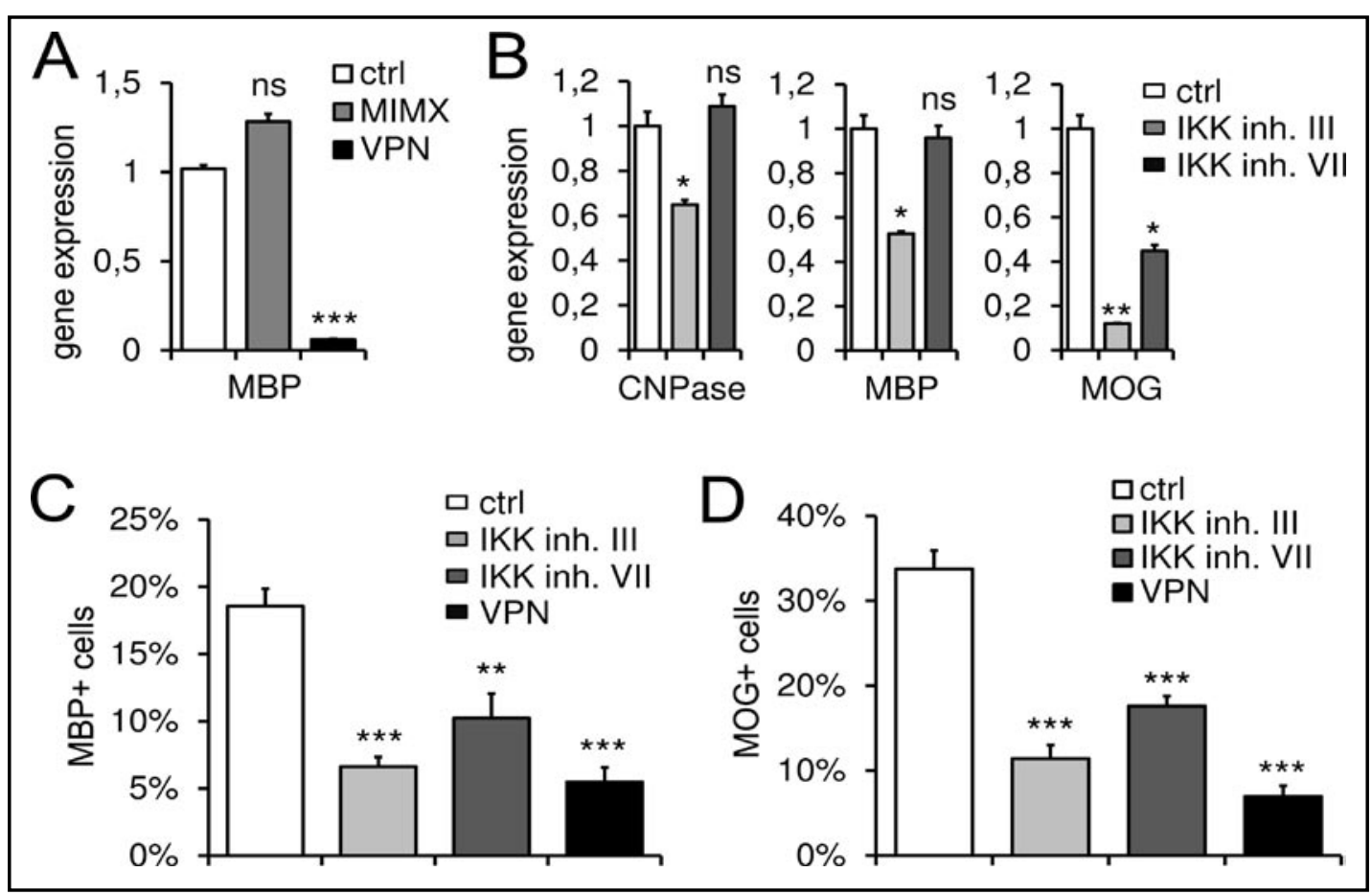

Fig. 7. Regulation of myelin expression in oligodendroglial precursor cells. (A) In contrast to VPN the PDE1 specific inhibitor MIMX (applied as $10 \mu \mathrm{M}$ concentration for a duration of three days) failed in reducing MBP transcript levels. (B) Analysis of CNPase, MBP and MOG gene expression in OPCs treated for three days with either $0.5 \mu \mathrm{M}$ IKK inhibitor III or $0.25 \mu \mathrm{M}$ IKK inhibitor VII. Whereas IKK inhibitor III reduced expression levels of all three myelin genes the IKK inhibitor VII exerted only a significant effect on MOG transcript levels. GAPDH expression was used as reference. One representative out of three independent measurements is shown for each determination; data are mean values +/- SEM (Student's t-test of treatment vs. control at any time point: ${ }^{*} \mathrm{p}<0.05,{ }^{* *} \mathrm{p}<0.01,{ }^{* * *} \mathrm{p}<0.001$ ). (C) Quantitative determination of MBP- (C) and MOGpositive cells (D) upon application of IKK inhibitors for three and six days, respectively. Similar to transcript levels IKK inhibitor III reduced the degree of MBP- and MOG positive cells, whereas IKK inhibitor VII was less potent. One representative out of three independent measurements is shown; data are mean values +/SEM (Student's t-test: ns: not significant; ${ }^{* *} \mathrm{p}<0.01,{ }^{* * *} \mathrm{p}<0.001$ ).

of CXCL12, indicating that VPN interferes with spontaneous as well as chemokine promoted oligodendroglial differentiation.

\section{Oligodendroglial differentiation inhibitors are induced by vinpocetine}

In light of the observed strong negative impact of VPN on oligodendroglial differentiation we investigated whether this effect was mediated via activation of so called oligodendroglial differentiation inhibitors, a group of genes that has been related to the impairment of OPC differentiation and myelin repair [7]. OPCs were stimulated with VPN and on days one, three, six and nine cells were lysed and gene expression profiles of 18 different OPC differentiation inhibitors were measured. Real-time qRT-PCR was performed for genes encoding catenin- $\beta 1$, Hes1, Hes5, Id2, Id4, Lingo1, MKP5, NLGN3, Notch1, p57kip2, PKC, ptprj, PTPRR, RhoA, Rock2, TIP30, Trpc4 and Tspan2. This analysis revealed that out of these genes, the transcriptional regulators Id2, Id4, and Hes1 and the serine/threonine kinase Rock2 showed an increase in their expression over time (Fig. 6) whereas the expression of the p57kip2 gene [22] was first inhibited by VPN and then induced at later time points. 
Fig. 8. Gene expression analysis of negative regulators in oligodendroglial precursor cells differentiation over time in response to IKK inhibitor application. OPCs were treated with IKK inhibitors III, VII or control buffer and transcript levels were determined on days one, three, six and nine by means of real-time quantitative RTPCR. Both IKK inhibitors induced the expression of Id2, Id4 and Hes1. GAPDH expression was used as reference. One representative out of three independent measurements is shown; data are mean values +/SEM (Student's t-test of treatment vs. control at any time point: ns: not significant; $\left.{ }^{*} \mathrm{p}<0.05 ;{ }^{* * *} \mathrm{p}<0.001\right)$.

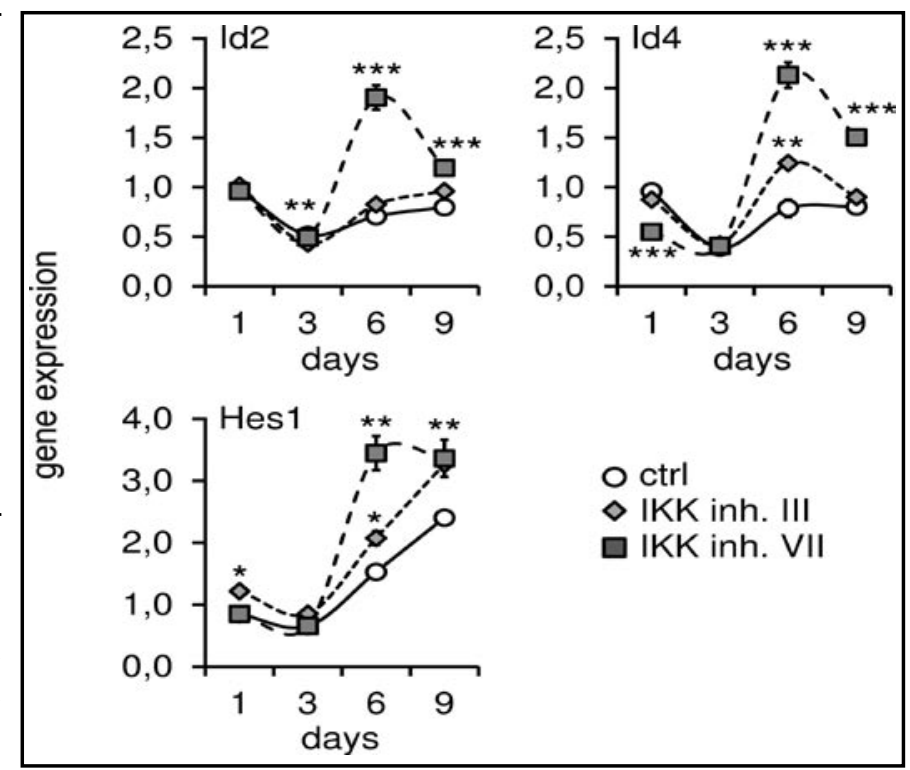

$I \kappa B$ kinase inhibitors can mimic vinpocetine dependent differentiation effects

VPN can act as specific inhibitor of PDE1 and was shown to increase cAMP and cGMP levels in some cells [26]. To find out whether the differentiation inhibitory effect exerted by VPN was based on changes of cyclic nucleotide levels we determined cAMP and cGMP levels applying specific enzyme linked immunosorbent assays (ELISA, Enzo Life Sciences, Lörrach, Germany). Interestingly, no differences in cAMP or cGMP levels were observed between VPN stimulated and control buffer treated OPCs at a number of different time points (data not shown), whereas forskolin treatment (as positive control) resulted in significantly increased cAMP levels (data not shown). Likewise the application of another PDE1 specific blocker 8-methoxymethyl-3-isobutyl-1-methylxanthine (MIMX; applied at different concentrations and studied at different time points) was not able to reproduce myelin gene suppression but let to a non-significant increase of MBP levels (Fig. 7A). This strongly suggested that VPN acts via its recently published ability to suppress IKB kinase (IKK; [12]). We therefore treated OPCs with two well described IKK blockers, IKK inhibitor III (4-(2'-aminoethyl)amino-1,8dimethylimidazo[1,2-a]quinoxaline) and VII (benzamido-pyrimidine), and determined CNPase, MBP and MOG gene expression levels after different time points. While IKK inhibitor III significantly reduced expression of all three myelin genes (shown after 72 hours), IKK inhibitor VII was only able to reduce MOG expression (Fig. 7B). OPCs treated for three and six days with these IKK inhibitors were then immunostained and the degree of MBP- and MOG-positive cells was determined. This demonstrated that IKK inhibitor III treatment also led to significantly diminished myelin protein expression whereas IKK inhibitor VII application negatively affected both myelin markers to a lesser degree (Fig. 7C, D). Finally, we wanted to know whether the IKK inhibitors were also able to regulate the expression of oligodendroglial differentiation inhibitors (Fig. 8). This demonstrated that similar to VPN treated cells, both IKK inhibitors could augment the expression of genes encoding Id2, Id4 and Hes1, whereas p57kip2 and Rock2 expression profiles were not severely changed (data not shown). In addition, VPN also induced Hes5 transcript levels at day 3 (data not shown), a negative regulatory gene that was not affected by VPN. These observations provide additional evidence that in OPCs VPN acts primarily as an inhibitor of IKB kinase and by doing so strongly interferes with morphological maturation and myelin expression. 


\section{Discussion}

PDE inhibitors have been proposed for MS treatment based on their ability to ameliorate clinical signs and inflammation in EAE animals [16-18]. But little is known about PDE mediated effects on neural cells or regenerative processes such as remyelination. As several PDEs could be involved in the regulation of intracellular levels of cyclic nucleotides [27], we investigated whether VPN (PDE1 inhibitor), CTZ, (PDE3 inhibitor), RLP (PDE4 inhibitor) or combinations of these inhibitors affected OPC maturation processes. Our studies revealed that among the substances tested VPN exerted a strong and reproducible negative effect on OPC differentiation. Importantly, CTZ and RLP did not regulate myelin expression or morphological maturation, suggesting a unique effect of VPN. This is in line with previous observations where RLP combined with lovastatin was applied to EAE animals and was shown to provide neuroprotection and to facilitate neurorepair [21]. However, neuroregenerative effects were only detected with the combined medication, but not following single treatment with RLP. Furthermore, in an in vivo model of spinal cord damage, CTZ was shown to increase the number of oligodendrocytes in the damaged areas [28], probably due to enhanced cell migration, but no evidence for a direct role on myelin repair could be obtained. Of note the negative regulatory effect of VPN was not restricted to spontaneous OPC differentiation, since we could show that a strong differentiation promoting stimulus such as provided by CXCL12 dependent CXCR7 activation [23] was also completely abolished in presence of this substance. This suggests that VPN either interferes with several signal transduction pathways or that it interacts with a centrally placed regulator.

Following our observations that VPN did not elevate intracellular levels of cAMP and cGMP and given that the structurally unrelated PDE1 specific inhibitor MIMX was unable to reproduce the negative effect on oligodendroglial differentiation, we concluded that VPN was likely to act through blockade of IKB kinase (IKK) activity as previously shown [12]. This mode of action was confirmed using two unrelated IKK inhibitors, both of which had a negative impact on myelin expression and also induced several oligodendroglial differentiation inhibitors such as Id2, Id4 or Hes1. It remains to be elucidated whether these three inhibitory transcriptional regulators solely account for the negative effects exerted by VPN (and the IKK inhibitors) and to what degree p57kip2 and Rock2 are functionally implicated in the VPN dependent process. Likewise it is currently not known whether the quantitative differences seen upon application of VPN and the two IKK inhibitors result from true functional differences or are due to different binding capacities or pharmacological kinetics. Based on our observation we can nevertheless conclude that in oligodendroglial precursor cells VPN is likely to block IKK which in turn can no longer phosphorylate IкB and hence remains stable and active $[29,30]$. When bound to NFKB it interferes with its translocation to the nucleus and therefore NFKB cannot act as transcriptional activator, assuming that $\mathrm{NF} \kappa \mathrm{B}$ exerts a similar regulatory function in oligodendroglial cells as recently shown for the Schwann cell lineage [31].

IKK has been related to immunosuppressive as well as neuroprotective effects in an EAE model [32]. Analysis of IKK2 knock-out mice revealed that IKK2 is not required for developmental myelination, but that cuprizone mediated demyelination was less severe in IKK2 depleted- as compared to wild type mice [33]. To our knowledge, only one other IKK inhibitor (AS602868) has been tested in oligodendrocytes [34], but results were ascribed to survival and cell excitotoxicity. Of note, it has also been reported that Hes1 expression in cancer cells is related to IKK2 [35], but how IKK inhibition and induction of negative transcriptional regulators are related in oligodendroglial cells still needs to be addressed in future studies.

PDE inhibitors acting as anti-inflammatory agents have been suggested as potential MS treatments. While further studies on direct suppression of IKK by VPN in the oligodendroglial context as well as the demonstration of a direct negative effect on remyelination in vivo are still needed, our data provide the rationale to restrict its application to patients with de- or dysmyelinating conditions. Caution is advised not only in patients with progressive 
Torres/Göttle/Kremer et al.: IкB Kinase and Oligodendrocyte Maturation

disease courses, but also in a remitting-relapsing multiple sclerosis (RRMS) given that intra-individual differences among patients might make it difficult to clearly discriminate between active inflammatory, degenerative and regenerative phases. Therefore, in light of VPN's widespread use as nutritional supplement, supposed to confer increased brain health and function, it is worth evaluating whether such a potential general health benefit is indeed outweighing the risk of a disturbed endogenous myelin repair process.

\section{Acknowledgements}

We thank Brigida Ziegler for preparation and culture of primary OPCs. This work was supported by a McDonald fellowship to K.T. provided by the Multiple Sclerosis International Foundation (MSIF) and a grant of the Gemeinnützige Hertie-Stiftung (to P.K.). The MS Center at the Department of Neurology is supported in part by the Walter-and-Ilse Rose Stiftung.

\section{References}

1 Aktas O, Küry P, Kieseier B, Hartung HP: Fingolimod is a potential novel therapy for multiple sclerosis. Nat Rev Neurol 2010;6:373-382.

2 Taveggia C, Feltri ML, Wrabetz L: Signals to promote myelin formation and repair. Nat Rev Neurol 2010;6:276-287.

3 Gallo V, Armstrong RC: Myelin repair strategies: a cellular view. Curr Opin Neurol 2008;21:278-283.

4 Menn B, Garcia-Verdugo JM, Yaschine C, Gonzalez-Perez O, Rowitch D, Alvarez-Buylla A: Origin of oligodendrocytes in the subventricular zone of the adult brain. J Neurosci 2006;26:7907-7918.

5 Nait-Oumesmar B, Picard-Riera N, Kerninon C, Decker L, Seilhean D, Hoglinger GU, Hirsch EC, Reynolds R, Baron-Van Evercooren A: Activation of the subventricular zone in multiple sclerosis: evidence for early glial progenitors. Proc Natl Acad Sci U S A 2007;104:4694-4699.

-6 Fancy SP, Kotter MR, Harrington EP, Huang JK, Zhao C, Rowitch DH, Franklin RJ: Overcoming remyelination failure in multiple sclerosis and other myelin disorders. Exp Neurol 2010;225:18-23.

7 Kremer D, Aktas 0, Hartung HP, Küry P: The complex world of oligodendroglial differentiation inhibitors. Ann Neurol 2011;69:602-618.

8 Domek-Lopacinska K, Strosznajder JB: Cyclic GMP metabolism and its role in brain physiology. J Physiol Pharmacol 2005;56 :S15-34.

9 Medina AE: Therapeutic utility of phosphodiesterase type I inhibitors in neurological conditions. Front Neurosci 2011;5:21.

10 Kureshiro J, Miyamoto K, Tanaka N, Kusunoki S: Selective phosphodiesterase-3 inhibitor cilostazol ameliorates experimental autoimmune encephalomyelitis. Neuroreport 2009;20:718-722.

11 Paintlia AS, Paintlia MK, Singh I, Singh AK: Combined medication of lovastatin with rolipram suppresses severity of experimental autoimmune encephalomyelitis. Exp Neurol 2008;214:168-180.

12 Jeon KI, Xu X, Aizawa T, Lim JH, Jono H, Kwon DS, Abe J, Berk BC, Li JD, Yan C: Vinpocetine inhibits NFkappaB-dependent inflammation via an IKK-dependent but PDE-independent mechanism. Proc Natl Acad Sci U S A 2010;107:9795-9800.

13 Medina AE: Vinpocetine as a potent antiinflammatory agent. Proc Natl Acad Sci U S A 2010;107:9921-9922.

14 Patyar S, Prakash A, Modi M, Medhi B: Role of vinpocetine in cerebrovascular diseases. Pharmacol Rep 2011;63:618-628.

15 McDaniel MA, Maier SF, Einstein GO: "Brain-specific” nutrients: a memory cure? Nutrition 2003;19:957975.

16 Chen GQ, Chen YY, Wang XS, Wu SZ, Yang HM, Xu HQ, He JC, Wang XT, Chen JF, Zheng RY: Chronic caffeine treatment attenuates experimental autoimmune encephalomyelitis induced by guinea pig spinal cord homogenates in Wistar rats. Brain Res 2010;1309:116-125.

17 Pifarre P, Prado J, Baltrons MA, Giralt M, Gabarro P, Feinstein DL, Hidalgo J, Garcia A: Sildenafil (Viagra) ameliorates clinical symptoms and neuropathology in a mouse model of multiple sclerosis. Acta Neuropathol 2011;121:499-508. 


\section{Cellular Physiology Cell Physiol Biochem 2012;30:711-722 and Biochemistry \\ Torres/Göttle/Kremer et al.: IкB Kinase and Oligodendrocyte Maturation}

18 Dinter H, Tse J, Halks-Miller M, Asarnow D, Onuffer J, Faulds D, Mitrovic B, Kirsch G, Laurent H, Esperling P, Seidelmann D, Ottow E, Schneider H, Tuohy VK, Wachtel H, Perez HD: The type IV phosphodiesterase specific inhibitor mesopram inhibits experimental autoimmune encephalomyelitis in rodents. J Neuroimmunol 2000;108:136-146.

19 Genain CP, Roberts T, Davis RL, Nguyen MH, Uccelli A, Faulds D, Li Y, Hedgpeth J, Hauser SL: Prevention of autoimmune demyelination in non-human primates by a cAMP-specific phosphodiesterase inhibitor. Proc Natl Acad Sci U S A 1995;92:3601-3605.

-20 Sommer N, Loschmann PA, Northoff GH, Weller M, Steinbrecher A, Steinbach JP, Lichtenfels R, Meyermann R, Riethmuller A, Fontana A, et al.: The antidepressant rolipram suppresses cytokine production and prevents autoimmune encephalomyelitis. Nat Med 1995;1:244-248.

-21 Paintlia AS, Paintlia MK, Singh I, Skoff RB, Singh AK: Combination therapy of lovastatin and rolipram provides neuroprotection and promotes neurorepair in inflammatory demyelination model of multiple sclerosis. Glia 2009;57:182-193.

22 Kremer D, Heinen A, Jadasz J, Göttle P, Zimmermann K, Zickler P, Jander S, Hartung HP, Küry P: p57kip2 is dynamically regulated in experimental autoimmune encephalomyelitis and interferes with oligodendroglial maturation. Proc Natl Acad Sci U S A 2009;106:9087-9092.

-23 Göttle P, Kremer D, Jander S, Odemis V, Engele J, Hartung HP, Küry P: Activation of CXCR7 receptor promotes oligodendroglial cell maturation. Ann Neurol 2010;68:915-924.

-24 Dinter H: Phosphodiesterase type 4 inhibitors: potential in the treatment of multiple sclerosis? BioDrugs 2000;13:87-94.

25 Miron VE, Kuhlmann T, Antel JP: Cells of the oligodendroglial lineage, myelination, and remyelination. Biochim Biophys Acta 2011;1812:184-193.

-26 Guerra de Gonzalez L, Gonzalez de Alfonzo R, Lippo de Becemberg I, Alfonzo MJ: Cyclic nucleotidedependent phosphodiesterases (PDEI) inhibition by muscarinic antagonists in bovine tracheal smooth muscle. Biochem Pharmacol 2004;68:651-658.

-27 Cheng J, Grande JP: Cyclic nucleotide phosphodiesterase (PDE) inhibitors: novel therapeutic agents for progressive renal disease. Exp Biol Med (Maywood) 2007;232:38-51.

-28 Beaumont E, Whitaker CM, Burke DA, Hetman M, Onifer SM: Effects of rolipram on adult rat oligodendrocytes and functional recovery after contusive cervical spinal cord injury. Neuroscience 2009;163:985-990.

29 Bonizzi G, Karin M: The two NF-kappaB activation pathways and their role in innate and adaptive immunity. Trends Immunol 2004;25:280-288.

-30 Perkins ND: Integrating cell-signalling pathways with NF-kappaB and IKK function. Nat Rev Mol Cell Biol 2007;8:49-62.

-31 Morton PD, Johnstone JT, Ramos AY, Liebl DJ, Bunge MB, Bethea JR: Nuclear factor-kappaB activation in Schwann cells regulates regeneration and remyelination. Glia 2012;60:639-650.

-32 Emmanouil M, Taoufik E, Tseveleki V, Vamvakas SS, Tselios T, Karin M, Lassmann H, Probert L: Neuronal I kappa B kinase beta protects mice from autoimmune encephalomyelitis by mediating neuroprotective and immunosuppressive effects in the central nervous system. J Immunol 2009;183:7877-7889.

-33 Raasch J, Zeller N, van Loo G, Merkler D, Mildner A, Erny D, Knobeloch KP, Bethea JR, Waisman A, Knust M, Del Turco D, Deller T, Blank T, Priller J, Bruck W, Pasparakis M, Prinz M: IkappaB kinase 2 determines oligodendrocyte loss by non-cell-autonomous activation of NF-kappaB in the central nervous system. Brain 2011;134:1184-1198.

-34 Sarnico I, Boroni F, Benarese M, Alghisi M, Valerio A, Battistin L, Spano P, Pizzi M: Targeting IKK2 by pharmacological inhibitor AS602868 prevents excitotoxic injury to neurons and oligodendrocytes. J Neural Transm 2008;115:693-701.

-35 Maniati E, Bossard M, Cook N, Candido JB, Emami-Shahri N, Nedospasov SA, Balkwill FR, Tuveson DA, Hagemann T: Crosstalk between the canonical NF-kappaB and Notch signaling pathways inhibits Ppargamma expression and promotes pancreatic cancer progression in mice. J Clin Invest 2011;121:46854699. 